\title{
Improving Probabilistic Ensemble Forecasts of Convection through the Application of QPF-POP Relationships
}

\author{
CHRistopher J. SchafFeR* AND William A. GALlus JR. \\ Department of Geological and Atmospheric Sciences, Iowa State University, Ames, Iowa \\ Moti SEGAL \\ Department of Agronomy, Iowa State University, Ames, Iowa
}

(Manuscript received 24 May 2010, in final form 2 January 2011)

\begin{abstract}
Four new approaches of postprocessing quantitative precipitation forecasts (QPFs) from model ensemble output were used to generate probability of precipitation (POP) tables in order to develop a forecasting method that could outperform a traditional method that relies upon calibration of POP forecasts derived using equal weighting of ensemble members. Early warm season 10-member ensemble output from the NOAA Hazardous Weather Testbed Spring Experiments was used, with 29 cases serving as a training set to create the POP tables and 20 cases used as a test set. The new approaches use QPF-POP relationships based on two properties termed precipitation amount characteristic (PAC) and ensemble member agreement. Exploratory results are presented for $20-\mathrm{km}$ grid spacing and selectively for $4-\mathrm{km}$ grid spacing. In the first approach, POPs were based on a binned PAC and the number of ensemble members with 6-h precipitation accumulations greater than given thresholds. In a second approach, a neighborhood method was used to find the number of points in a given neighborhood area around each of the domain grid points with precipitation amounts greater than a given threshold, while also considering the binned PAC representative of the neighborhood. A third approach synthesized the previous methods and led to an increase in skill relative to the individual methods, and a fourth approach using a combination of methods produced forecasts with even greater skill. All of the forecasts from the four approaches were improved statistically significantly compared to the calibrated traditional method's forecasts at $20-\mathrm{km}$ grid spacing. The second approach on its own showed skill comparable to that obtained by a traditional calibrated 10 -member ensemble, so adopting this approach alone could potentially save computer resources that could then be used for model refinements, only sacrificing the increased skill that could have been obtained by using the fourth approach.
\end{abstract}

\section{Introduction}

Ensemble forecasts have many advantages over deterministic forecasts. Ensemble forecasts facilitate probabilistic forecasts and provide a direct measure of uncertainty, unlike deterministic forecasts. Ensemble forecasts are more useful than single deterministic forecasts because small errors in a single forecast's initial conditions will grow exponentially over time, making the deterministic forecast increasingly unreliable (Hamill and Colucci 1997).

\footnotetext{
* Current affiliation: National Weather Service, Goodland, Kansas.

Corresponding author address: Christopher J. Schaffer, National Weather Service, 920 Armory Rd., Goodland, KS 67735.

E-mail: christopher.schaffer@noaa.gov
}

Also, ensemble mean forecasts tend to be more skillful than any single member forecast (Leith 1974; Ebert 2001; Chakraborty and Krishnamurti 2006).

Probabilities of precipitation (POPs) can be derived from ensemble forecasts in a variety of ways. Most simply, POPs are determined by considering the percentage of ensemble members forecasting precipitation greater than a specified threshold amount. For a 10-member ensemble with equal weighting assigned to each member, the forecast POPs would be $0 \%, 10 \%, 20 \%$, up to $100 \%$. In this study, this method will be referred to as the uncalibrated traditional method (Uncali_trad, hereafter), because it is the simplest approach to determining POPs (Hamill and Whitaker 2006). Hamill and Colucci (1997) showed how calibration using observed data can improve POPs created using a Gumbel distribution fit to ensemble data, while Hamill and Whitaker (2006) described a 


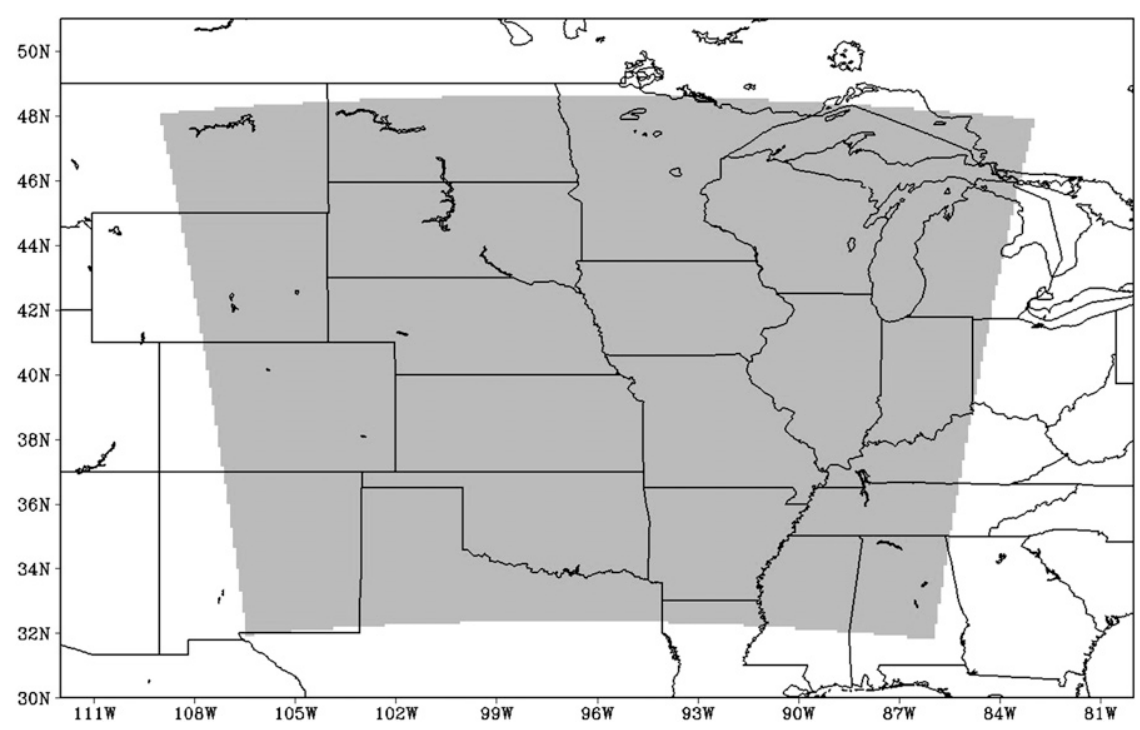

FIG. 1. Subdomain (shaded) over which the POP forecasting approaches were tested.

method to calibrate POPs using reforecasts. A calibrated version of the traditional method (Cali_trad hereafter) formed by training over observed data can be used to provide improved forecasts, helping to correct for some biases.

It is hypothesized in the present study that more elaborate methods, using single or ensemble model output, can be used to obtain POP forecasts that are potentially superior to those from Cali_trad and Uncali_trad. For instance, separating quantitative precipitation forecasts (QPFs) into precipitation "bins" can provide new ways of obtaining useful probabilistic information (e.g., Gallus and Segal 2004, hereafter GS04; Gallus et al. 2007, hereafter GBE07; Yussouf and Stensrud 2008). Recently, various studies used a neighborhood approach, which in its simplest form considers an area surrounding a grid point in order to gain POP improvements by accounting for spatial precipitation probability constraints (e.g., Theis et al. 2005; Ebert 2008; Roberts and Lean 2008; Ebert 2009; Gilleland et al. 2009; Schwartz et al. 2010). Operational centers have also begun using techniques like spatial density plots that incorporate neighborhood approaches (D. Novak, National Centers for Environmental Prediction, 2010, personal communication). Using unique precipitation data available for the central United States for the domain depicted in Fig. 1, the present study generally presents an exploratory attempt to identify variants of such approaches to provide gridpoint-related POPs that outperform the aforementioned traditional approaches. Previous studies have not addressed this specific objective.

GS04 and GBE07 used a precipitation-binning method in a deterministic forecast (denoted hereafter as GSD) to show that, at grid points where the "binned" amount of forecasted precipitation was larger, the probability that those grid points would receive at least a small amount of precipitation was greater than where the forecasted precipitation amount was smaller. They attributed this to the fact that when the models predicted larger amounts of precipitation, the atmospheric state was such that precipitation was more likely to occur. In GS04, it also was noted that POP values increased even further if two different models showed an intersection of grid points with precipitation in a specified bin. The GS04 findings suggest that the QPF-POP relationship might yield an even better forecast if the relationship was applied to ensemble forecasts.

The specific goal of this study is to identify and apply postprocessing approaches similar to the GS04 and neighborhood approaches, as well as a hybrid of both, to traditional model ensemble forecasts, and to examine how the resulting POPs compare to those from traditional approaches. Section 2 describes the general methodology, modeling and data aspects, and the new approaches. Section 3 presents the results from different postprocessing methods and provides Brier scores, ROC areas, and additional features that will be used when comparing methods to the more traditional approaches. Discussion and conclusions follow in section 4.

\section{Methodology and data}

\section{a. General methodology}

The new approaches of determining POPs in the present study mostly involved the creation of $2 \mathrm{D}$ POP tables (termed POP tables hereafter) based on parameters related to the following two properties: (i) the forecasted 
TABLE 1. Brief descriptions and abbreviations of the various methods used in the study and their classification within the adopted approaches. An $N_{0}$ indicates the optimized $N$ value, i.e., for which the minimum BS was obtained.

\begin{tabular}{|c|c|c|c|}
\hline Approach/method & First parameter $(\mathrm{P} 1)$ & Second parameter $(\mathrm{P} 2)$ & Parameter values \\
\hline \multicolumn{4}{|c|}{ (a) Reference approaches } \\
\hline $\begin{array}{l}\text { GSD (see GS04 and } \\
\text { GBE07) }\end{array}$ & Binned QPF & - & $\mathrm{P} 1=7$ \\
\hline Uncali_trad & - & Uncalibrated traditional ensemble agreement & $\mathrm{P} 2=10$ \\
\hline Cali_trad & - & Calibrated traditional ensemble agreement & $\mathrm{P} 2=10$ \\
\hline $\begin{array}{l}\text { Simplified version of } \\
\text { Theis et al. (2005) }\end{array}$ & - & Uncalibrated spatial ensemble agreement & $\begin{array}{l}\mathrm{P} 2=\mathrm{N} \times \mathrm{N} \\
N_{0}=21\end{array}$ \\
\hline Binary & - & $\begin{array}{l}\text { POP }=100 \% \text { if forecast } \geq \text { a threshold, } \\
\text { and } 0 \% \text { otherwise }\end{array}$ & - \\
\hline \multicolumn{4}{|c|}{ (b) Two-parameter point approach } \\
\hline Max_thr & Binned QPF (max) & Calibrated traditional ensemble agreement & $\mathrm{P} 1=7, \mathrm{P} 2=10$ \\
\hline Ave_thr & Binned QPF (avg) & Calibrated traditional ensemble agreement & $\mathrm{P} 1=7, \mathrm{P} 2=10$ \\
\hline \multicolumn{4}{|c|}{ (c) Two-parameter neighborhood approach } \\
\hline Max_nbh & Binned QPF (max) & Calibrated spatial ensemble agreement & $\begin{array}{l}\mathrm{P} 1=7, \mathrm{P} 2=N \times N, \\
N_{0}=13\end{array}$ \\
\hline Ave_nbh & Binned QPF (avg) & Calibrated spatial ensemble agreement & $\begin{array}{l}\mathrm{P} 1=7, \mathrm{P} 2=N \times N, \\
N_{0}=15\end{array}$ \\
\hline \multicolumn{4}{|c|}{ (d) Two-parameter neighborhood- $M$ approach } \\
\hline Ave_nbh-M & Binned QPF (avg) & $\begin{array}{l}\text { Calibrated traditional and spatial } \\
\text { ensemble agreement }\end{array}$ & $\begin{array}{l}\mathrm{P} 1=7, M=10 \\
\mathrm{P} 2=N \times N \times M \\
N_{0}=11\end{array}$ \\
\hline \multicolumn{4}{|c|}{ (e) Combination approach } \\
\hline Combination & Various & Various & - \\
\hline
\end{tabular}

precipitation amount characteristic (PAC) within a bin (as in GS04) and (ii) the number of ensemble members forecasting agreement on the occurrence of precipitation above a threshold amount (as traditionally used for ensemble-based POP forecasts). In this paper, the term ensemble will not only refer to the traditional definition of sets of model forecasts as defined previously but will also be used for a number of related grid points within an area surrounding each grid point of the domain (earlier termed neighborhood). The term method will refer to a variant of an approach.

The POPs in the tables were assigned by finding the correct alarm ratio (referred to as the hit rate in GS04) for each case in the training dataset. The correct alarm ratio is defined as $h / f$, where $f$ is the number of grid points with precipitation forecasted for a given combination of bin and member agreement (such a combination is termed a scenario) and $h$ is the number of "hits," or points where the observed precipitation also exceeded the specified threshold.

In the present study, the first of the above two POP table properties (the PAC) is given at any grid point by either taking the maximum forecasted amount from any ensemble member, or by taking the ensemble average. Seven precipitation bins were used (with units of inches that are commonly used operationally; $1 \mathrm{in} .=25.4 \mathrm{~mm}$ ), including $<0.01,0.01-0.05,0.05-0.10,0.10-0.25,0.25-0.50$, $0.50-1.00$, and $>1.0$. Considering the GS04 conclusion that points with larger QPFs had a greater likelihood of receiving precipitation relative to points with smaller QPFs, and considering the higher deterministic skill for the ensemble-averaged precipitation field compared to any member, these two properties are likely to yield a POP table with improved forecasting skill. Using a PAC was necessary because each of the ensemble members provides a precipitation amount, and a single representative precipitation amount was needed at each grid point to apply the binning approach as used by GS04. Conceptually, POP tables can be of higher dimensions if additional variants of the properties and related parameters are considered. Detailed descriptions of the various forecasting approaches in this study and their applications are provided in section $2 \mathrm{~d}$ and summarized in Table 1. However, a brief description of the general concepts and features along with some illustrations should be useful to the reader and is outlined in the remainder of this subsection.

The concepts introduced previously for establishing new POP approaches are similar to those widely used for traditional ensemble models. They differ, however, in the specific way that the POP is generated. Table 2 provides the probability for the various combinations of bin sorting for maximum precipitation among the 10 ensemble members (i.e., PAC), and the percentage of agreement for precipitation exceeding given thresholds. The probabilities in Table 2, generated during the 
TABLE 2. POP table (\%) for the 0.01-in. threshold in the Max_thr method with the corresponding number of grid points in parentheses. Top row designates the accumulated precipitation bin, and the left-side column shows the percentage of ensemble members that forecasted precipitation $>0.01$-in. threshold; "ave" stands for average.

\begin{tabular}{|c|c|c|c|c|c|c|c|c|}
\hline \multirow{2}{*}{$\begin{array}{c}\text { Ensemble } \\
\text { agreement (\%) }\end{array}$} & \multicolumn{8}{|c|}{ Bin range (in.) } \\
\hline & $<0.01$ & $0.01-0.05$ & $0.05-0.10$ & $0.10-0.25$ & $0.25-0.50$ & $0.50-1.0$ & $>1.0$ & Column ave \\
\hline \multirow[t]{2}{*}{0} & 2.8 & - & - & - & - & - & - & 2.8 \\
\hline & (721 837) & $(0)$ & $(0)$ & $(0)$ & (0) & (0) & (0) & (721 837) \\
\hline \multirow[t]{2}{*}{10} & - & 11.4 & 15.4 & 18.1 & 18.6 & 19.7 & 28.7 & 12.8 \\
\hline & $(0)$ & $(80102)$ & (13683) & (8873) & (2904) & (1091) & (369) & (107 022) \\
\hline \multirow[t]{2}{*}{20} & - & 14.3 & 19.2 & 22.3 & 23.8 & 26.7 & 31.3 & 18 \\
\hline & $(0)$ & $(36475)$ & $(15360)$ & (13010) & (4929) & (2192) & (803) & $(72769)$ \\
\hline \multirow[t]{2}{*}{30} & - & 16.1 & 23.5 & 26.2 & 30.5 & 30.6 & 39.1 & 23.4 \\
\hline & $(0)$ & (18532) & (13338) & $(14282)$ & $(6516)$ & (3383) & (1385) & $(57436)$ \\
\hline \multirow[t]{2}{*}{40} & - & 18.5 & 25 & 31.5 & 36.3 & 39.9 & 39.9 & 29.3 \\
\hline & (0) & $(10081)$ & (10863) & $(14403)$ & (7969) & (4367) & (1872) & $(49555)$ \\
\hline \multirow[t]{2}{*}{50} & - & 19.4 & 27.6 & 36 & 42.5 & 45.8 & 46.8 & 35.5 \\
\hline & $(0)$ & (5282) & (8388) & (13593) & (8815) & (5411) & (2479) & (43968) \\
\hline \multirow[t]{2}{*}{60} & - & 19.7 & 28.3 & 39.3 & 47.4 & 52.9 & 55.3 & 41.6 \\
\hline & (0) & (2901) & (6379) & $(12615)$ & (9379) & (6671) & (3504) & $(41449)$ \\
\hline \multirow[t]{2}{*}{70} & - & 23 & 31.4 & 42.5 & 53.1 & 57 & 61.7 & 47.9 \\
\hline & $(0)$ & (1821) & (4927) & (11463) & (10318) & (7998) & (4459) & (40 986) \\
\hline \multirow[t]{2}{*}{80} & - & 21.5 & 33 & 47.2 & 56.9 & 63.3 & 66.3 & 54.5 \\
\hline & (0) & (922) & (3588) & $(10510)$ & (11461) & (9931) & (5987) & (42 399) \\
\hline \multirow{2}{*}{90} & - & 15.8 & 35.4 & 53.6 & 66.8 & 71.4 & 77.6 & 65.5 \\
\hline & $(0)$ & (438) & (2792) & (10506) & $(14840)$ & $(14738)$ & (10196) & $(53510)$ \\
\hline \multirow[t]{2}{*}{100} & - & 16.8 & 27.6 & 55.2 & 73.3 & 83.9 & 89.2 & 78.6 \\
\hline & (0) & (167) & (1642) & (9954) & $(21333)$ & (30 985) & (25 648) & (89729) \\
\hline \multirow[t]{2}{*}{ Row ave } & 2.8 & 13.7 & 23.7 & 36.6 & 53.1 & 65.6 & 74.5 & 19.4 \\
\hline & (721 837) & $(156721)$ & $(80960)$ & (119209) & (98464) & $(86767)$ & $(56702)$ & $(1320660)$ \\
\hline
\end{tabular}

training stage, are based on the aggregated contribution of all simulated domain points for the various cases and 6-hourly periods. This POP table reflects a two-parameter space (Fig. 2a). Using the POP table to issue a POP forecast, the combination of binned PAC and ensemble member agreement (i.e., scenario) determines the POP for each given point of the simulation domain.

Figure $2 \mathrm{a}$ also represents the general format for POP tables, in which the boxes each represent a unique forecast scenario from the bin and agreement parameter spaces. Based on the description above, the probability given in the rhs column of Table 2 (column average) provides the calibrated POP for various member agreements (Cali_trad). On the other hand, a weighted member-average POP for each bin is given by the bottom row (row average) probability. The one-parameter GSD approach used in GS04 and GBE07 also provided POPs for each bin, but it did not use ensemble data. The neighborhood approaches (see segments $\mathrm{c}$ and $\mathrm{d}$ in Table 1) have POP tables similar to Table 2 , except that the ensemble members are spatially generated and typically have far more than 10 members.

\section{b. Modeling and data aspects}

Ensemble forecast output for the early warm season in the central United States was generated by the 2007 and
2008 National Oceanic and Atmospheric Administration (NOAA) Hazardous Weather Testbed Spring Experiments, which took place during April-June of both years (Kong et al. 2007; Xue et al. 2008). The ensemble consisted of 10 members using the Advanced Research module of the Weather Research Forecast Model (WRF-ARW) with 4-km grid spacing run by the Center for Analysis and Prediction of Storms (CAPS) located at the University of Oklahoma. The experiments differed somewhat between the first and second years. In the 2007 experiment, 4 of the 10 members used both perturbed initial conditions and mixed physical parameterizations, and the remaining 6 members (including the control member) used only mixed physical parameterizations. In the 2008 experiment, 8 of the 10 members used both perturbed initial conditions and mixed physical parameterizations. Descriptions of the initial conditions and lateral boundary conditions used can be found in Kong et al. (2007) and Xue et al. (2008). The 2007 experiment was initialized at 2100 UTC, while the 2008 experiment was initialized at 0000 UTC. Because of the differences in initialization time, the first $3 \mathrm{~h}$ of the 2007 data were excluded for each day, and five 6-h accumulated precipitation periods, 0000-0600, 0600-1200, 1200-1800, 1800-0000, and 0000-0600 UTC, were used to create the POP forecasts. The POP tables used in this study are time 
(a)

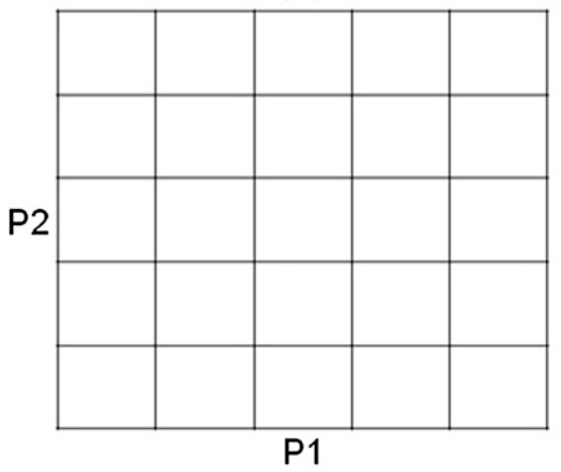

(b)

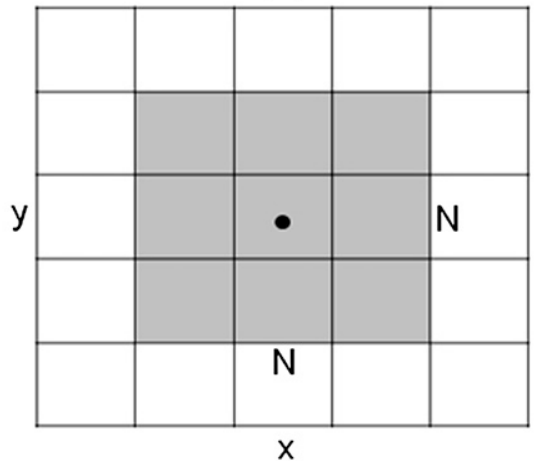

(c)

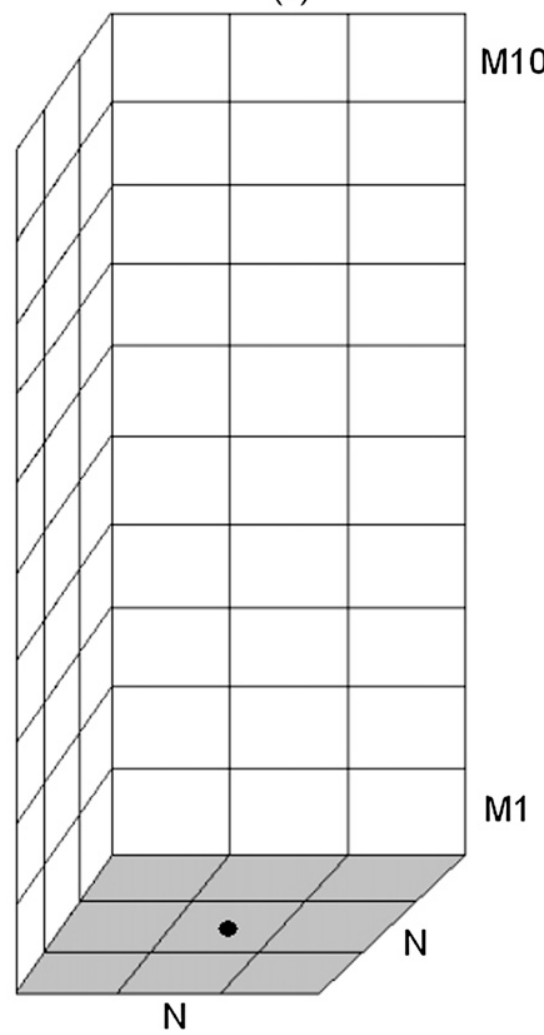

FIG. 2. Schematic illustration related to the two-parameter forecast approaches. See Table 1 for complementary information. (a) A two-parameter space where each square represents a scenario defined by a combination of discrete values of P1 (representing binned PACs) and P2 (representing member agreement percentage), (b) a simulated domain $(x, y)$ with a grid point (dotted) surrounded by an $N \times N$ neighborhood (shaded) providing the discrete values for the parameters P1 and P2, and (c) an $N \times N \times M$ neighborhood- $M$ to be used in the $x-y$ domain of the simulations providing the discrete values for the parameters $\mathrm{P} 1$ and $\mathrm{P} 2(M=10$, in the present study).

independent, however, because forecasts from all time periods were grouped together to increase the sample size used for calibration in the correct alarm ratio calculations.

The 2008 output was also on a larger grid than the 2007 output $(3600 \mathrm{~km} \times 2700 \mathrm{~km}$ versus $3000 \mathrm{~km} \times$ $2500 \mathrm{~km}$ ), but the present study uses the subdomain (Fig. 1) used in Clark et al. (2009) with a size of $1980 \mathrm{~km} \times$ $1840 \mathrm{~km}$. National Centers for Environmental Prediction (NCEP) stage IV precipitation analysis (Baldwin and Mitchell 1997) was used to designate hits at a forecast point if the observed rainfall amount was greater than a threshold. Compared to gauge-only observations, stage IV data can have a moist bias at thresholds less than $0.50 \mathrm{in}$. in $24 \mathrm{~h}$ and a dry bias at thresholds greater than 0.50 in. (Schwartz and Benjamin 2000). The stage IV data, along with the forecasts, were interpolated to grids using NCEP procedures that conserve the total amount of liquid in the domain.

\section{c. Issues influencing the presentation of results}

Two grid spacings were considered on this subdomain: (i) a $20-\mathrm{km}$ grid generated by mapping the $4-\mathrm{km}$ output from both years to the new domain and (ii) the original $4-\mathrm{km}$ grid. Our probabilistic analysis focuses on the $20-\mathrm{km}$ output because these coarser grid spacings are used in operational ensembles at present. Additionally, the averaging to a $20-\mathrm{km}$ grid effectively removes noise in the forecasted precipitation features associated with short wavelengths, and the averaging of the observed precipitation yields a field compatible with the numerical damping of predicted fields for short waves of $\leq 7 \Delta x$ (e.g., Tustison et al. 2001; Skamarock 2004). Gallus (2002), among others, showed that deterministic traditional skill measures are generally better for coarser grid spacings than finer ones, whereas analyzing 4-km data requires greater computational and time resources. Given the above arguments, sensitivity tests were performed selectively using the 4-km output. 
For both grid spacings, the POP tables were created from the 29 cases from 2008, and were tested against the 20 cases from 2007. Sensitivity tests of training over the 2007 cases and testing against the 2008 cases showed no major differences, so the results were not shown. It is possible the changes stated above in the ensemble design between the two years would have some impact on the performance of the approaches presented, but this lack of sensitivity to year used for training and testing implies the impacts are small. For each method, the probability forecasts were verified using decomposed Brier scores (BSs), Brier skill scores (BSSs), bias calculations, and relative operating characteristic (ROC) areas. Reliability diagrams, ROC diagrams, BS scatterplots, and additional illustrations of skill were examined. Differences were tested for statistical significance using paired $t$ tests of BSs for all cases and time periods. Paired $t$ tests were chosen because of the paired nature of the datasets and because this test is especially useful when the correlation and sample size are high. The brief summary of the methods tested and their classification into several approaches given in Table 1 should be useful in reading the paper. If not stated otherwise, the presented results are valid for $20-\mathrm{km}$ grid spacing. Finally, results were presented in tables as needed to obtain better insight into the results. The four new approaches created and tested for this study are described in detail in the next section.

\section{d. New forecast approaches}

\section{1) TWO-PARAMETER POINT APPROACH}

The first forecasting approach analyzed made use of two parameters: the 6-h period PAC (denoted P1) and the percentage/number of ensemble members forecasting precipitation above a given threshold (denoted P2), both determined at each grid point from the 10-member ensemble model output. It consists of a two-parameter space illustrated in Fig. 2a with parameter discretization values of $\mathrm{P} 1=7$ and $\mathrm{P} 2=10$. It is worth noting that the GSD approach could be represented in the figure as just one row of boxes in Fig. 2a. Two definitions of the PAC were used when testing this approach, so two POP tables for each threshold were created, in methods denoted as Max_thr [its related POP table (Table 2) provides various quantified details related to the above two-parameter space] and Ave_thr. An example of how a POP would be assigned from Max_thr at a given grid point is as follows: if the maximum precipitation amount from the 10 ensemble members is $0.29 \mathrm{in}$., and 8 of the 10 members are forecasting precipitation above the 0.01-in. threshold, then both the bin (0.25-0.50 in.) and agreement ( $80 \%)$ have been determined and a POP can be assigned from the 0.01-in. threshold Max_thr POP table (in this example, $56.9 \%)$.

\section{2) TWO-PARAMETER NEIGHBORHOOD APPROACH}

A second forecasting approach was developed using neighborhood methods, as described briefly in the Introduction. Specifically, it is a $2 \mathrm{D}$ neighborhood approach that can be thought of in more than one way. For instance, considering a square neighborhood with $(N \times$ $N)$ grid points centered at domain grid point $(I, J)$, then each of the $(N \times N)$ sets of grid points with the same relative orientation to the domain grid points $(I, J)$ may be considered ensemble members. Alternatively, the neighborhood ensemble may be viewed as generated by a displacement of the simulated domain grid $(N \times N)$ times relative to the domain original grid points $(I, J)$ while the displaced points delineate an $(N \times N)$ square around $(I, J)$. Hence, for an example using $N=3$, the ensemble effectively represents the original grid (no displacement) and eight displacements of the simulated domain by one grid point north, west, east, south, southeast, southwest, northeast, and northwest.

In the present study, within a specified square area around a center point representing a neighborhood (a $N \times N$ gridpoint area, where $N$ is an odd integer; see illustration in Fig. 2b), the maximum or average PAC was determined and placed in a bin. A square area was used instead of a circular area for the sake of simplicity, as the shape of the neighborhood has little impact on the results (Ebert 2009). If a neighborhood intersected the domain's boundary, the agreement parameter was calculated as shown in the appendix. It is worth noting that specifying a $1 \times 1$ point "area" reduces the approach to binning precipitation at a single point (i.e., to the GSD approach).

This approach uses two parameters to generate POP tables: the binned PAC (corresponding to P1 in Fig. 2a) and the member agreement percentage, which provides the percentage of members/points within the neighborhood with forecast precipitation amounts greater than a given threshold (P2). The Max_thr and similar methods considered forecasts from 10 ensemble members, but because this neighborhood approach (abbreviated as Max_nbh or Ave_nbh) uses each of the points within the neighborhood, all of these points can be thought of as a spatially generated pseudo-ensemble (e.g., Theis et al. 2005). This neighborhood approach was applied to deterministic QPFs because it uses a spatial ensemble instead of using the 10 WRF-ARW members as an ensemble (a traditional ensemble). For this reason, the approach was used on each of the 10 WRF-ARW members. Different POP tables were created by increasing the neighborhood size 
for each of the 10 members until the optimal size $\left(N_{0} \times N_{0}\right.$, corresponding to the lowest BS) was determined. In the 10 WRF-ARW members used in this study, there is a systematic difference in skill and other characteristics for each ensemble member, meaning the outcome of each member is not equally likely.

An example of how a POP would be assigned from Ave_nbh at a center grid point in the Fig. $2 b$ neighborhood (with $N=3$ ) is as follows: if the average precipitation amount from the nine neighborhood members is $0.29 \mathrm{in}$., and eight of the nine members are forecasting precipitation above the 0.01 -in. threshold, then both the bin (0.25-.50 in.) and agreement ( $88.9 \%)$ have been determined and a POP can be assigned from the 0.01-in. threshold Ave_nbh POP table for $N=3$ (table not shown).

This approach is different than the fractions skill score (FSS) method tested in Schwartz et al. (2010), which issued POP forecasts for entire neighborhood areas. In the present study the POP forecasts are for individual grid points. It is worth noting, however, that Schwartz et al. (2010) found that POPs as good as those of the uncalibrated traditional forecasts exist based upon FSS evaluations.

\section{3) TWO-PARAMETER NEIGHBORHOOD- $M$ APPROACH}

A third forecasting approach considered both definitions of ensemble members from the previous two approaches, the $10 \mathrm{WRF}-\mathrm{ARW}$ members, and the $N \times N$ spatial ensemble members, when determining the PAC and the member agreement parameter. Hence, a 3D neighborhood consisting of $N \times N \times M$ members (termed hereafter neighborhood- $M ; M=10$ in the present study) was formed. To establish a PAC, this approach averages the forecast precipitation amounts from all of the $N \times N \times M$ members associated with each of the simulation domain grid points. An illustration of a 3D neighborhood- $M$ can be seen in Fig. 2c. Each cube represents an ensemble member that would be considered in the binning and member agreement parameters, so that $N \times N \times 10$ members were considered for each of the simulation domain grid points.

For large neighborhoods, the number of possible forecast scenarios would become very large, and this could have a negative impact on the efficiency of the approach. By introducing too many forecast scenarios, the grid points considered in the correct alarm ratio statistics could become overdispersed, which would degrade the approach's reliability. To decrease the number of forecast scenarios, members considered in the member agreement parameter were grouped such that 10 consecutive members were placed in each group. By grouping members in this way, $N \times N$ general member groups, each containing 10 specific members, could be considered, rather than considering $N \times N \times 10$ specific members. For example, if anywhere from 20 to 29 members from the $3 \times 3 \times 10$ threedimensional neighborhood depicted in Fig. $2 c$ had precipitation greater than a threshold, these 10 specific members would be considered as part of one of 9 general member groups.

\section{4) COMBinAtion APPROACH}

A final forecasting approach was examined that combined several of the previous methods by averaging their POPs. Considering each contributing method as an ensemble member that consists itself of ensemble members, this approach can be viewed as a "superensemble" generated by postprocessing. Because POP fields over the domain for the different methods evidenced forecast spread, it was hypothesized that averaging the POPs of multiple methods might result in a forecast superior to the individual methods. This hypothesis was also supported by the common finding that an ensemble mean forecast tends to be more skillful than any single member forecast.

\section{Results}

\section{a. Two-parameter point forecast approach}

\section{1) POP TABLES}

An illustrative POP table created for the 0.01-in. observed precipitation threshold using the Max_thr method is shown in Table 2. Due to space constraints, tables for the 0.10- and 0.25-in. thresholds, as well as tables for Ave_thr, are not shown but can be found as a supplement online (http://www.meteor.iastate.edu/ schaffec/poptables.html). As the PAC increased, the POP tended to increase for each of the three thresholds.

As the percentage of ensemble members with precipitation amounts greater than the threshold (a traditional way of defining POPs from ensembles) increased, the POPs also generally increased. In the few instances where POPs decreased with an increasing percentage of members, such as for the lowest bins, there were relatively few points associated with the percentage calculation, which may have accounted for the unusual behavior. The increase in POPs associated with both increasing PAC and ensemble member agreement percentage resulted in the highest POPs (lower-right corner of the table). The high POPs indicate that precipitation was almost inevitable when most or all members forecasted heavy amounts. Conversely, a combination of low PACs and low member agreement percentages yielded low POPs (upper-left side of the table). Grid points contributing to the second column of Table 2 are restricted by definition of the method; if the maximum PAC was less than 0.01 in. (essentially no precipitation), then all members had accumulated precipitation less than the lowest threshold. This definition 
(a)

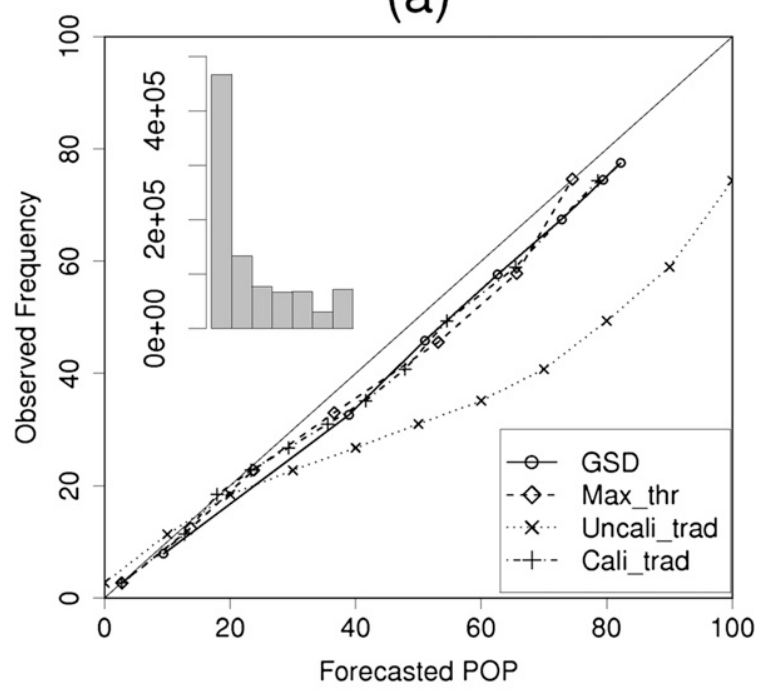

(b)

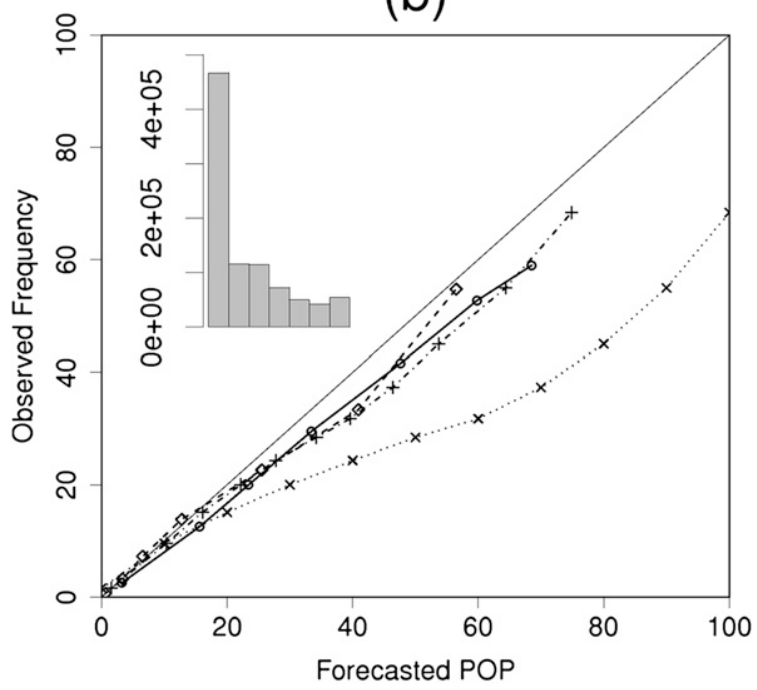

(c)

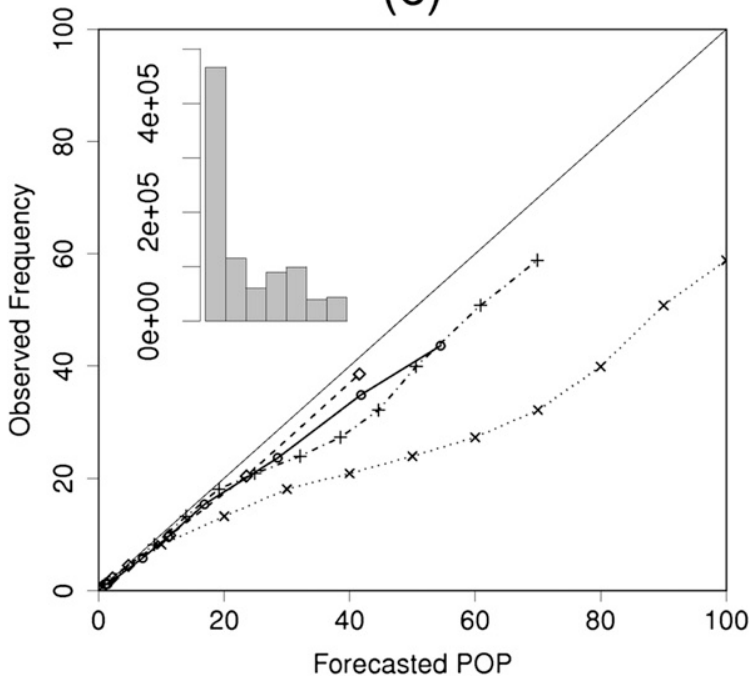

results in a very low POP value for this scenario, which is fitting because we would expect a very low likelihood of precipitation when none of the ensemble members are forecasting measurable precipitation.

The right-most column of Table 2 uses a weighted summation over all bins for each member agreement percentage, indicating what the POP would be for each member percentage if binning of the PAC was not considered. These POPs increase with increasing member agreement percentage and are the values used for Cali_trad. In addition, Cali_trad can be thought of as a traditional method (denoted Uncali_trad, defined as equally weighted forecasts yielding POPs of $0 \%, 10 \%, 20 \%$, etc., for this 10 -member ensemble) that has been adjusted using observations, and as the conditional POP given the ensemble agreement across all bins. By definition, Max_thr and Ave_thr provide a refinement of Cali_trad.

The bottom row in Table 2 is a weighted summation over all member percentages. This row provides a single POP representative of each precipitation bin, like the GSD approach. The POPs increase with increasing bin amounts. These POPs determined the bins of probability used when making reliability and ROC diagrams, because they allow for bin-representative points on the diagrams, as found in the diagrams in GS04 and GBE07.

\section{2) RELIABILITY DIAGRAMS}

By testing against the independent 2007 dataset, it was clear that the reliability of Uncali_trad was poorer at all three thresholds (Fig. 3) than that for Max_thr, Cali_ trad, and a forecast applying the previous GSD oneparameter precipitation-binning method described in the introduction to one of the 10 ensemble members for comparison purposes. There was similar reliability among Max_thr, Cali_trad, and GSD. As the threshold increases, Max_thr and GSD show slightly better reliability than Cali_trad.

\section{3) BRIER SCORES}

BSs were examined to quantitatively compare POP forecasts among the methods. The BS is defined as

FIG. 3. Reliability diagrams for GSD, Max_thr, Uncali_trad, and Cali_trad at thresholds of (a) 0.01 , (b) 0.10 , and (c) 0.25 in. Histogram displays the distribution of Max_thr forecasts within the seven bins described in section 2, with the first bin for precipitation frequency $<0.01 \mathrm{in}$. and the last bin at the right for $>0.50 \mathrm{in}$. The seven bins are associated with the following POPs (\%): (a) 2.8, 13.7, 23.7, 36.6, 53.1, 65.6, and 74.5; (b) 0.6, 3.3, 6.6, 12.8, 25.5, 40.9, and 56.5 ; and (c) $0.2,1.2,2.2,4.7,11.3,23.6$, and 41.5 . 


$$
\mathrm{BS}=\frac{1}{n} \sum_{k=1}^{n}\left(p_{k}-o_{k}\right)^{2},
$$

where $p_{k}$ is the forecast probability for forecast $k$ of $n$ total forecasts and $o_{k}$ is the observed probability (either $0 \%$ or $100 \%$ ) corresponding to each forecast. Using the method described by Murphy (1973) and Wilks (2006), BSs can be decomposed into three components: reliability, resolution, and uncertainty. The decomposition is mathematically described as

$$
\mathrm{BS}=\frac{1}{n} \sum_{i=1}^{I} N_{i}\left(p_{i}-\bar{o}_{i}\right)^{2}-\frac{1}{n} \sum_{i=1}^{I} N_{i}\left(\bar{o}_{i}-\bar{o}\right)^{2}+\bar{o}(1-\bar{o}),
$$

where

$$
\begin{aligned}
& \bar{o}_{i}=\frac{1}{N_{i}} \sum_{k \in N_{i}} o_{k} \text { and } \\
& \bar{o}=\frac{1}{n} \sum_{i=1}^{I} N_{i} \bar{o}_{i},
\end{aligned}
$$

where $N_{i}$ is the number of forecasts in the $i$ th forecast category and $n$ is the total number of forecasts. The first, second, and third terms on the right-hand side of Eq. (2) represent the reliability, resolution, and uncertainty components of the Brier score, respectively. The reliability component, like in reliability diagrams, compares forecasts to observed frequencies, while the resolution component quantifies how well a method discerns different types of events. The uncertainty component is independent of the forecast approach used because it only considers observations. BSs are essentially a measure of the mean squared probability error, so smaller scores (preferably close to 0 ) are ideal. For this reason, a small reliability component and a large resolution component are desired. The decomposed BSs in all approaches/methods except the combination approach were calculated from POPs explicitly, and did not use bins of probability, such as those used when making the reliability and ROC diagrams. Depending on the computational resources available and method complexity, either decomposition technique can be used to calculate the BS as expressed in Eq. (1).

Brier skill scores were also computed:

$$
\mathrm{BSS}=\frac{\mathrm{BS}-\mathrm{BS}_{\mathrm{ref}}}{\mathrm{BS}_{\mathrm{per}}-\mathrm{BS}_{\mathrm{ref}}}=1-\frac{\mathrm{BS}}{\mathrm{BS}_{\mathrm{ref}}},
$$

where the reference $\mathrm{BS}$ (denoted $\mathrm{BS}_{\mathrm{ref}}$ ) is the sample climatology and the perfect $\mathrm{BS}$ (denoted $\mathrm{BS}_{\mathrm{per}}$ ) is 0 . When calculating the sample climatology, $o$ is used for $p_{i}$ in the decomposition equation [Eq. (2)], so the reference BS is reduced to the uncertainty. Large BSSs indicate better skill compared to the sample climatology. Finally, a bias statistic was also calculated, using

$$
\text { bias }=\frac{\sum_{k=1}^{n} p_{k}}{\sum_{k=1}^{n} o_{k}} .
$$

Table 3 shows the overall decomposed BSs at each threshold for the new methods, and for comparison purposes shows GSD, Uncali_trad, Cali_trad, and a reference score based on a binary forecasting method (using POPs of either $100 \%$ or $0 \%$, denoted "Binary"). Instead of showing each of the 10 GSD and Binary forecasts, the results in the table are the averaged results for the 10 (GSD_ave10 and Binary) and best 5 (GSD_ave5) members. The best five GSD members represent a "well chosen" ensemble that does not vary over cases, and is based only on the BSs for the 10 GSD members. For all thresholds, the BSs for the new methods were always smaller (closer to zero) than the GSD, Binary, and Uncali_ trad BSs. As thresholds increase, however, the degree by which the scores differ tends to decrease. In all cases Max_thr and Ave_thr had higher BSSs and lower bias scores than GSD, Binary, and Uncali_trad. When compared to the Cali_trad BSs, Max_thr and Ave_thr still have more favorable scores.

The $p$ values from the paired $t$ tests of the 100 BSs (20 cases with five time periods each) for each method showed that the Max_thr results were statistically significantly different at the $99.9 \%$ confidence level for the $0.01-, 0.10$-, and 0.25 -in. thresholds when compared to the Uncali_trad results, the best results from GSD (member 10), and the Cali_trad results with $p$ values consistently $<0.001$. The decomposed BS equation shows that a low BS can be obtained if the reliability and uncertainty terms are both small and the resolution term large. All of the newly presented methods using the two-parameter point forecast approach had larger resolution terms than GSD, Binary, Uncali_trad, and Cali_trad. Of all the methods Cali_trad had the smallest reliability term. The reliability diagrams (Fig. 3) clearly showed that Uncali_trad had worse reliability than the other methods, which the reliability component of the Brier decomposition confirmed. Finally, the uncertainty term decreased with increasing thresholds, but it did not differ between methods because the uncertainty is only a function of the sample climatology and is thus independent of forecast method.

The bias values for all methods showed an overestimation in the POP forecasts, though Max_thr and Ave_thr had values closest to 1 , showing more favorable 
TABLE 3. Decomposed BSs, BSSs, bias scores, and ROC areas for the two new methods of the two-parameter point forecast approaches, the GSD 10-member and best 5-member averages, Uncali_trad, and Cali_trad at thresholds of 0.01, 0.10, and 0.25 in.

\begin{tabular}{|c|c|c|c|c|c|c|c|}
\hline \multirow[b]{2}{*}{ Method } & \multicolumn{7}{|c|}{ Score } \\
\hline & BS & Reliability & Resolution & Uncertainty & BSS & Bias & ROC \\
\hline \multicolumn{8}{|l|}{0.01 in. } \\
\hline Binary & 0.1930 & 0.0766 & 0.0293 & 0.1456 & -0.3250 & 1.4707 & 0.712 \\
\hline GSD_ave10 & 0.1175 & 0.0073 & 0.0354 & 0.1456 & 0.1932 & 1.3488 & 0.763 \\
\hline GSD_ave5 & 0.1133 & 0.0063 & 0.0386 & 0.1456 & 0.2219 & 1.3234 & 0.777 \\
\hline Uncali_trad & 0.1234 & 0.0257 & 0.0480 & 0.1456 & 0.1530 & 1.4707 & 0.861 \\
\hline Cali_trad & 0.1040 & 0.0064 & 0.0480 & 0.1456 & 0.2855 & 1.2609 & 0.862 \\
\hline Max_thr & 0.1013 & 0.0097 & 0.0540 & 0.1456 & 0.3041 & 1.2501 & 0.857 \\
\hline Ave_thr & 0.1013 & 0.0095 & 0.0538 & 0.1456 & 0.3041 & 1.2501 & 0.862 \\
\hline \multicolumn{8}{|l|}{0.10 in. } \\
\hline Binary & 0.1155 & 0.0512 & 0.0124 & 0.0767 & -0.5052 & 1.6305 & 0.704 \\
\hline GSD_ave10 & 0.0653 & 0.0046 & 0.0161 & 0.0767 & 0.1489 & 1.6043 & 0.800 \\
\hline GSD_ave5 & 0.0632 & 0.0041 & 0.0176 & 0.0767 & 0.1758 & 1.5696 & 0.816 \\
\hline Uncali_trad & 0.0705 & 0.0152 & 0.0214 & 0.0767 & 0.0810 & 1.6305 & 0.865 \\
\hline Cali_trad & 0.0593 & 0.0040 & 0.0214 & 0.0767 & 0.2267 & 1.4582 & 0.866 \\
\hline Max_thr & 0.0586 & 0.0059 & 0.0240 & 0.0767 & 0.2357 & 1.4192 & 0.877 \\
\hline Ave_thr & 0.0587 & 0.0058 & 0.0238 & 0.0767 & 0.2345 & 1.4405 & 0.865 \\
\hline \multicolumn{8}{|l|}{0.25 in. } \\
\hline Binary & 0.0743 & 0.0364 & 0.0050 & 0.0429 & -0.7316 & 1.9159 & 0.678 \\
\hline GSD_ave10 & 0.0386 & 0.0029 & 0.0072 & 0.0429 & 0.1006 & 1.9621 & 0.818 \\
\hline GSD_ave5 & 0.0377 & 0.0026 & 0.0078 & 0.0429 & 0.1205 & 1.9259 & 0.834 \\
\hline Uncali_trad & 0.0440 & 0.0105 & 0.0095 & 0.0429 & -0.0243 & 1.9159 & 0.854 \\
\hline Cali_trad & 0.0363 & 0.0028 & 0.0095 & 0.0429 & 0.1547 & 1.7572 & 0.854 \\
\hline Max_thr & 0.0359 & 0.0037 & 0.0108 & 0.0429 & 0.1633 & 1.6722 & 0.897 \\
\hline Ave_thr & 0.0358 & 0.0037 & 0.0108 & 0.0429 & 0.1655 & 1.6972 & 0.869 \\
\hline
\end{tabular}

biases relative to the other methods. While Max_thr and Ave_thr had the same bias value at the 0.01-in. threshold, the Max_thr method had a slightly better bias value than Ave_thr at the 0.10- and 0.25-in. thresholds.

\section{4) ROC DIAGRAMS}

ROC diagrams illustrate the ability of a forecast method to discern events and nonevents, while the areas under the curves (calculated using the trapezoidal method) quantify this discrimination. Both the diagrams and areas relate the probability of detection (POD) to the probability of false detection (POFD). An ideal ROC area is 1, with a curve that goes from the lower-left corner (where POD $=0$ and POFD $=0$ ) to the upperleft corner (where POD $=1$ and POFD $=0)$, and on to the upper-right corner (where POD $=1$ and POFD = 1). Figure 4 shows the ROC curves for Max_thr, GSD, Cali_trad, and Uncali_trad, while ROC areas for all methods are given in Table 3. Overall, the ROC areas were high; all values for all methods except Binary were greater than 0.70, which indicates a useful forecast (Buizza et al. 1999). Both Max_thr and Ave_thr, however, were also greater than the GSD values. The Cali_ trad and Uncali_trad ROC areas were higher than all other areas except Ave_thr at the 0.01-in. threshold, but the new methods had larger ROC areas than Cali_trad and Uncali_trad at the 0.25 -in. threshold (Max_thr was already larger than the Cali_trad and Uncali_trad ROC areas at the 0.10 -in. threshold). At the 0.01 -in. threshold Max_thr yielded a value of 0.857 , which was lower than the Ave_thr area at this threshold, and the Max_thr area increased to 0.897 at the 0.25 -in. threshold, which was higher than the related Ave_thr area. The increase in ROC areas shows that discrimination increased as the thresholds increased.

Both Max_thr and Ave_thr showed an increase in ROC area with increased thresholds. GS04 and GBE07 also noted this trend, which also occurred in the GSD method (Table 3). Both Cali_trad and Uncali_trad show a decrease in ROC area as thresholds increased, so the increased discrimination for forecasts of greater precipitation may be an added benefit of using the QPFPOP relationship compared to the more traditional approaches.

\section{b. Two-parameter neighborhood approach}

As discussed in section 2, the two-parameter neighborhood approach used spatially generated ensembles, rather than using the 10-member WRF-ARW ensemble like the two-parameter point forecast approach. For this reason, comparisons now must include the neighborhood size used $(3 \times 3$ grid points, $5 \times 5$, etc. $)$. Also, 
(a)

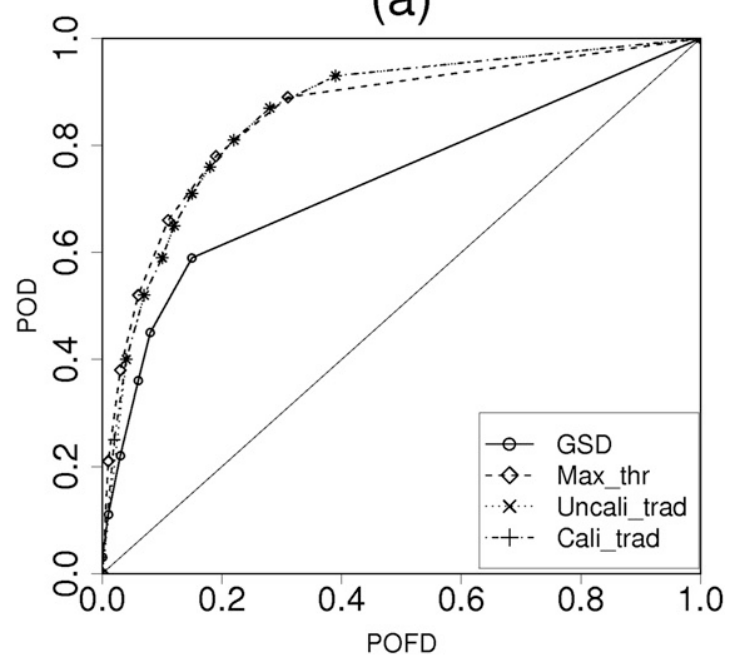

(b)

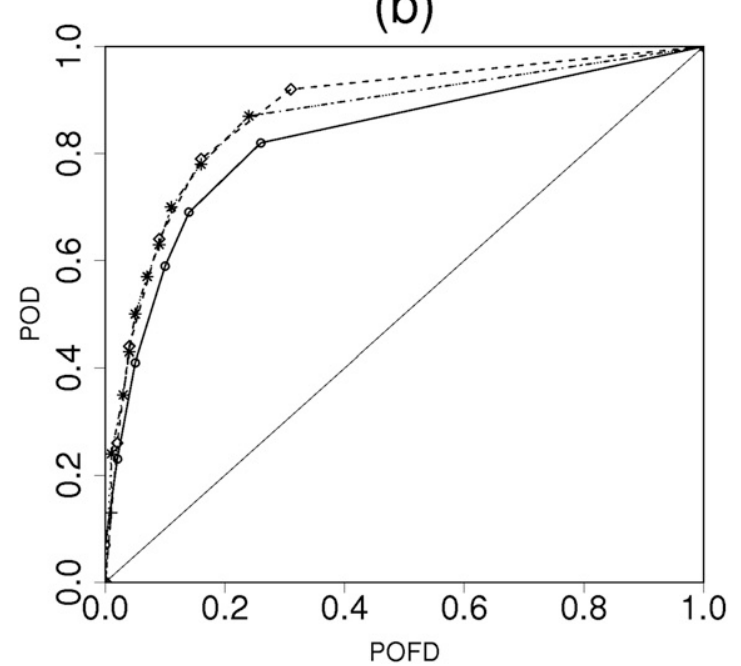

(c)

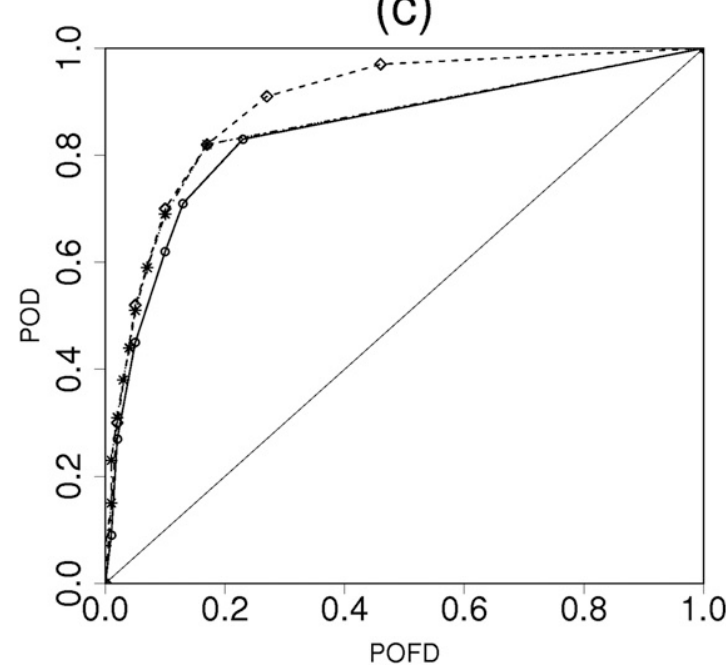

FIG. 4. The ROC diagrams for GSD, Max_thr, Uncali_trad, and Cali_trad at thresholds of (a) 0.01 , (b) 0.10 , and (c) $0.25 \mathrm{in}$. unlike the two-parameter point forecast approach, the two-parameter neighborhood approach was applied to deterministic forecasts, so BSs were generated for each of the 10 WRF-ARW members.

A $1 \times 1$ gridpoint "neighborhood," by definition of the two-parameter neighborhood approach's methodology, is a single point, so the BSs for Max_nbh and Ave_nbh matched those of the GSD method (shown in Table 3). As neighborhood size increased, the reliability of Max_nbh and Ave_nbh deteriorated, but resolution improved to a larger extent. The best BSs generally occurred for a $15 \times$ 15 point neighborhood for Ave_nbh (Table 4), after which the loss of reliability began to outweigh improvements in resolution. For Max_nbh, the best BSs occurred for a $13 \times$ 13 neighborhood (not shown). The best BSs for Ave_nbh, however, were lower (better) than the best scores for Max_nbh, suggesting that averaging the neighborhood points provides a more skillful forecast than selecting the maximum precipitation within the neighborhood.

The $15 \times 15$ Ave_nbh results (Table 4) showed that some BSs were greater than the Max_thr scores (Table 3), while others were less. For the 0.01-in. threshold, the lowest scores for Ave_nbh were below 0.1000, which was more skillful than the Max_thr, Ave_thr, and also Cali_ trad values. This result was surprising, because Max_thr, Ave_thr, and Cali_trad considered all 10 ensemble members when creating POPs, but Ave_nbh considered only an individual member. However, the neighborhood approach provided additional information so that POP forecasts made from single deterministic forecasts were comparable (or sometimes superior) to POP forecasts made using Cali_trad. The BSs of Ave_nbh applied to WRF-ARW member 8 (which yielded the best average BS in Table 4) were statistically significantly different from Cali_trad's scores at the $99.9 \%$ confidence level at all three thresholds, with $p$ values $<0.001$.

Theis et al. (2005) considered an uncalibrated spatiotemporal neighborhood approach in which the ratio of points in the neighborhood with precipitation above a threshold to the total number of points in the neighborhood determines the POP. The temporal component in Theis et al. (2005) considered 3-h time periods of precipitation accumulation. In the present study 6-h time periods were used, and because convective systems change substantially over 6-h periods, it was felt that the temporal neighborhood approach could not be used for the output available here. The simplified version of Theis et al. (2005) that considered only the spatial component provided maximum skill for a $21 \times 21$ gridpoint neighborhood and yielded a member-averaged Brier score of 0.1156 at the 0.01-in. threshold, so Ave_nbh provides better BSs compared to this method, as well. The simplified Theis results for a $15 \times 15$ gridpoint neighborhood and other 
TABLE 4. Decomposed BSs, BSSs, bias scores, and ROC areas for Ave_nbh $15 \times 15$ at thresholds of $0.01,0.10$, and 0.25 in. for the 10 simulated ensemble members and their average. The control member and members 6-10 used only mixed physics, while the remaining 4 members used mixed physics and perturbed initial conditions.

\begin{tabular}{|c|c|c|c|c|c|c|c|}
\hline \multirow[b]{2}{*}{ Member } & \multicolumn{7}{|c|}{ Score } \\
\hline & BS & Reliability & Resolution & Uncertainty & BSS & Bias & $\mathrm{ROC}$ \\
\hline \multicolumn{8}{|l|}{$0.01 \mathrm{in}}$. \\
\hline Mem1* & 0.1043 & 0.0279 & 0.0691 & 0.1456 & 0.2836 & 1.4890 & 0.862 \\
\hline Mem2 & 0.1113 & 0.0299 & 0.0642 & 0.1456 & 0.2354 & 1.4252 & 0.850 \\
\hline Mem3 & 0.1091 & 0.0261 & 0.0626 & 0.1456 & 0.2507 & 1.0603 & 0.829 \\
\hline Mem4 & 0.1102 & 0.0271 & 0.0625 & 0.1456 & 0.2430 & 1.1854 & 0.835 \\
\hline Mem5 & 0.1109 & 0.0280 & 0.0628 & 0.1456 & 0.2385 & 1.2827 & 0.836 \\
\hline Mem6 & 0.0990 & 0.0232 & 0.0699 & 0.1456 & 0.3203 & 1.1532 & 0.860 \\
\hline Mem7 & 0.1037 & 0.0270 & 0.0690 & 0.1456 & 0.2881 & 1.4137 & 0.863 \\
\hline Mem8 & 0.0988 & 0.0235 & 0.0703 & 0.1456 & 0.3218 & 1.1730 & 0.861 \\
\hline Mem9 & 0.0996 & 0.0239 & 0.0699 & 0.1456 & 0.3163 & 0.9587 & 0.861 \\
\hline Mem10 & 0.1007 & 0.0252 & 0.0701 & 0.1456 & 0.3085 & 1.3627 & 0.869 \\
\hline Ave & 0.1048 & 0.0262 & 0.0670 & 0.1456 & 0.2806 & 1.2504 & 0.853 \\
\hline \multicolumn{8}{|l|}{$0.10 \mathrm{in.}$} \\
\hline Mem1* & 0.0588 & 0.0140 & 0.0319 & 0.0767 & 0.2332 & 1.6641 & 0.895 \\
\hline Mem2 & 0.0644 & 0.0162 & 0.0286 & 0.0767 & 0.1611 & 1.5686 & 0.874 \\
\hline Mem3 & 0.0620 & 0.0136 & 0.0283 & 0.0767 & 0.1918 & 1.2104 & 0.860 \\
\hline Mem4 & 0.0629 & 0.0142 & 0.0279 & 0.0767 & 0.1798 & 1.3434 & 0.861 \\
\hline Mem5 & 0.0636 & 0.0149 & 0.0280 & 0.0767 & 0.1705 & 1.3901 & 0.862 \\
\hline Mem6 & 0.0572 & 0.0127 & 0.0322 & 0.0767 & 0.2543 & 1.3575 & 0.895 \\
\hline Mem7 & 0.0599 & 0.0149 & 0.0317 & 0.0767 & 0.2189 & 1.6494 & 0.895 \\
\hline Mem8 & 0.0576 & 0.0126 & 0.0318 & 0.0767 & 0.2497 & 1.3303 & 0.892 \\
\hline Mem9 & 0.0573 & 0.0125 & 0.0320 & 0.0767 & 0.2535 & 1.1045 & 0.890 \\
\hline Mem10 & 0.0577 & 0.0131 & 0.0321 & 0.0767 & 0.2482 & 1.4553 & 0.894 \\
\hline Ave & 0.0601 & 0.0139 & 0.0305 & 0.0767 & 0.2161 & 1.4074 & 0.882 \\
\hline \multicolumn{8}{|l|}{0.25 in. } \\
\hline Mem1* & 0.0356 & 0.0081 & 0.0154 & 0.0429 & 0.1717 & 1.9218 & 0.901 \\
\hline Mem2 & 0.0386 & 0.0098 & 0.0141 & 0.0429 & 0.1003 & 1.7731 & 0.885 \\
\hline Mem3 & 0.0367 & 0.0076 & 0.0138 & 0.0429 & 0.1446 & 1.3544 & 0.869 \\
\hline Mem4 & 0.0378 & 0.0082 & 0.0133 & 0.0429 & 0.1183 & 1.5411 & 0.870 \\
\hline Mem5 & 0.0381 & 0.0083 & 0.0132 & 0.0429 & 0.1134 & 1.6317 & 0.870 \\
\hline Mem6 & 0.0351 & 0.0076 & 0.0154 & 0.0429 & 0.1822 & 1.5685 & 0.906 \\
\hline Mem7 & 0.0367 & 0.0091 & 0.0153 & 0.0429 & 0.1442 & 1.9184 & 0.905 \\
\hline Mem8 & 0.0351 & 0.0075 & 0.0153 & 0.0429 & 0.1814 & 1.6095 & 0.902 \\
\hline Mem9 & 0.0350 & 0.0075 & 0.0155 & 0.0429 & 0.1847 & 1.3440 & 0.900 \\
\hline Mem10 & 0.0355 & 0.0080 & 0.0155 & 0.0429 & 0.1740 & 1.7059 & 0.905 \\
\hline Ave & 0.0364 & 0.0082 & 0.0147 & 0.0429 & 0.1515 & 1.6368 & 0.891 \\
\hline
\end{tabular}

* Control member.

neighborhood sizes less than $21 \times 21$ provided worse Brier scores than the $21 \times 21$ gridpoint BSs.

The bias values for the $10 \mathrm{WRF}-\mathrm{ARW}$ members to which Ave_nbh was most commonly applied showed forecast overestimation, which was seen with previous methods' bias values. The magnitude of the overestimation for some members was smaller than the overestimation experienced by Max_thr and Ave_thr. The member average bias value for Ave_nbh was slightly worse than the Max_thr and Ave_thr bias values at the 0.01-in. threshold, but the Ave_nbh average bias values were better (to a greater extent) at the 0.10 - and 0.25 -in. thresholds.

ROC areas for Ave_nbh again increased with increasing thresholds (Table 4). Many of the members had
ROC areas exceeding 0.90 at the 0.25 -in. threshold, which was an improvement over the previous methods' ROC areas. Improvement over Cali_trad can also be seen in scatterplots (Fig. 5) comparing the BSs of Ave_nbh applied to WRF-ARW member 8 at a $15 \times 15$ gridpoint neighborhood and Cali_trad's BSs, where each point is a BS comparison of the methods for a case and time. The majority of points are above the depicted identity line (diagonal), indicating that the Ave_nbh forecasts had lower BSs and thus higher skill than Cali_trad.

\section{c. Two-parameter neighborhood-M approach}

The two-parameter neighborhood- $M$ approach, like the two-parameter neighborhood approach in section $3 \mathrm{~b}$, 
(a)

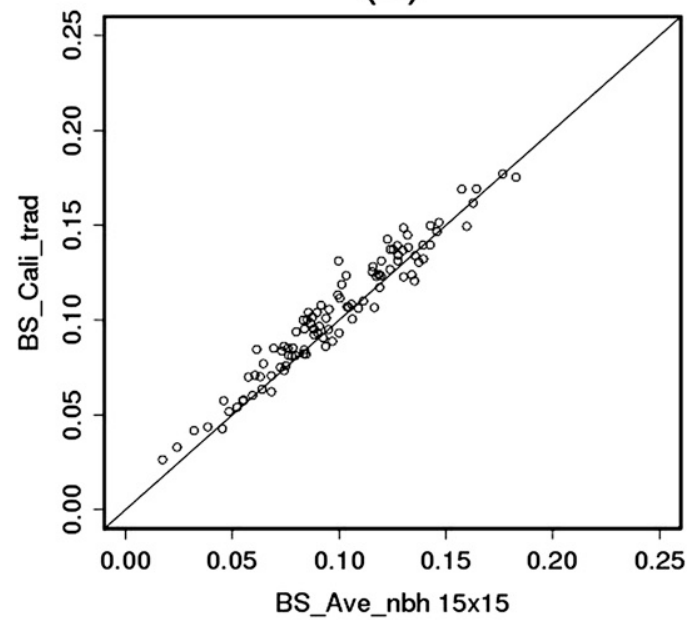

(b)

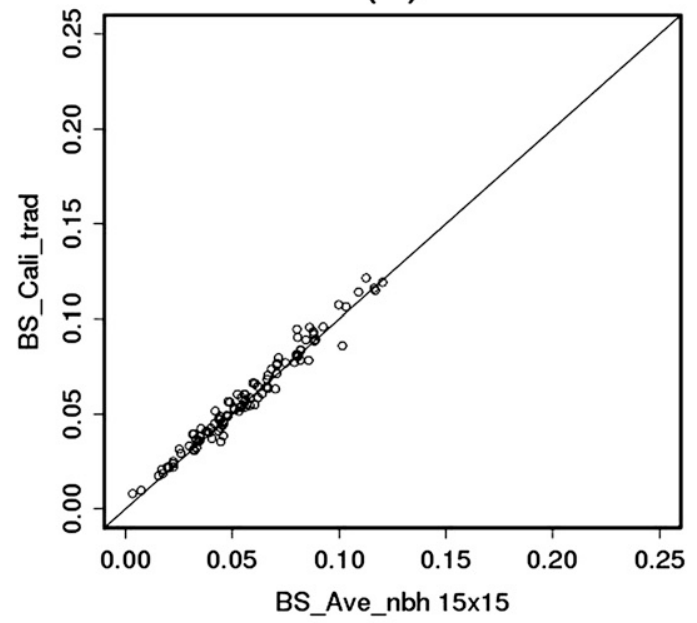

(c)

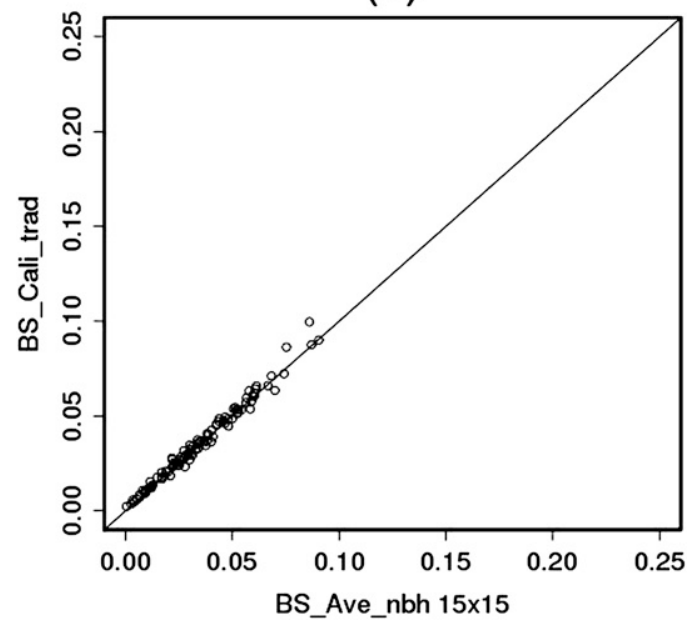

FIG. 5. Scatterplots of BSs for Ave_nbh (using ARW-WRF member 8 with a $15 \times 15$ neighborhood) and Cali_trad generated from the $100 \mathrm{BSs}$ from all cases and times at thresholds of (a) 0.01 , (b) 0.10 , and (c) 0.25 in. showed better skill for the average PAC, rather than the maximum PAC, so only the averaging version is presented. The two-parameter neighborhood- $M$ approach's BSs were best for an $11 \times 11$ point neighborhood (Table 5), were better than the BSs for the approaches in sections $3 \mathrm{a}$ and $3 \mathrm{~b}$, and were statistically significantly different from Cali_ trad's scores at the $99.9 \%$ confidence level at all three thresholds with all $p$ values $<0.001$ (and nearly 0 ). The bias values were better than the Max_thr, Ave_thr, and the member-averaged Ave_nbh bias values. The areas under the ROC curve for each threshold were larger than for Cali_trad and increased with increasing thresholds. The 0.01 -in.-threshold ROC area was 0.875 , and the 0.25 -in. area was 0.916 , which was larger than any of the previous methods' areas. The large ROC areas indicate that this approach discriminates better than do the approaches presented earlier.

\section{d. Combination approach}

By averaging the POPs for Ave_nbh, Max_thr, and Cali_trad, and increasing the Ave_nbh neighborhood size from $3 \times 3$ to $15 \times 15$, the BS improved from 0.0995 to 0.0959 for the 0.01 -in. threshold (Table 6). This is a relatively large improvement over the BS of 0.1014 associated with the Max_thr method. The POP forecasts were superior to any of the forecasts from other methods examined thus far. When compared to Cali_trad, the results for this combination approach were statistically significantly improved at the $99.9 \%$ confidence level with $p$ values $<0.001$ (and nearly 0 ) at all three thresholds. It should be noted that, while the combination approach's standard BSs from Eq. (1) and the decomposed BSs from Eq. (2) were identical, the BSs tested using the paired $t$ tests were generated from Eq. (1), so that the procedure for decomposition could not influence the statistics. The complexity of the combination approach required the use of bins of percentage instead of using POPs explicitly like in the other approaches. Bins of size $0.5 \%$ allowed the decomposed BSs to match the exact BSs from Eq. (1).

When the neighborhood for Ave_nbh within the combination approach was increased from $3 \times 3$ to $15 \times 15$ grid points, the reliability worsened, but the resolution improved to a greater extent. This pattern of behavior was also observed for Ave_nbh alone. The reliability was better for the ensemble of methods compared to Ave_nbh, however, likely due to the contribution of Max_thr and Cali_trad, which had better reliability scores than Ave_nbh at larger neighborhoods. Thus, the combination of methods had reliability comparable to Max_thr and resolution similar to Ave_nbh. By including the 10 forecasts from Ave_nbh, this combination approach used the ensemble average POP from 
TABLE 5. Decomposed BSs, BSSs, bias scores, and ROC areas for the two-parameter neighborhood- $M$ approach using $11 \times 11$ grid points at thresholds of $0.01,0.10$, and $0.25 \mathrm{in}$.

\begin{tabular}{cccccccr}
\hline \hline & \multicolumn{7}{c}{ Score } \\
\cline { 2 - 8 } Threshold & BS & Reliability & Resolution & Uncertainty & BSS & Bias & ROC \\
\hline 0.01 & 0.0965 & 0.0179 & 0.0670 & 0.1456 & 0.3371 & 1.2117 & 0.875 \\
0.10 & 0.0561 & 0.0097 & 0.0303 & 0.0767 & 0.2686 & 1.3371 & 0.903 \\
0.25 & 0.0346 & 0.0059 & 0.0142 & 0.0429 & 0.1935 & 1.5262 & 0.916 \\
\hline
\end{tabular}

the two-parameter neighborhood approach at each point, which alone yielded improved BSs compared to each of the individual Ave_nbh members. By including Ave_thr and the two-parameter neighborhood- $M$ approach in this combination approach, the skill increased marginally.

Though the improvements in BSs may sometimes appear small out of context, some are relatively large within the context of changes in BSs. BSs for precipitation forecasts are typically small numbers, and when averaging is done over a large dataset, differences in BSs among different approaches become even smaller. The change of 0.0036 between the values 0.0995 and 0.0959 is a relative change of almost $4 \%$. This change is comparable to or larger than those that often cause operational centers to change model codes. For instance, the Hydrometeorological Prediction Center (HPC) Verification Web site (http:// www.hpc.ncep.noaa.gov/html/hpcverif.shtml) has shown that a $4 \%$ change in BS for POPs is roughly one-third of the difference between average BSs for a day 3 forecast and a day 4 forecast, and is comparable to or larger than the HPC forecaster percentage improvement over model output statistics (MOS) forecasts for many months in 2009 and 2010 when forecasting for 12-h periods in the day 3-7 time frame. Finally, the new methods showing comparable or improved BSs compared to Cali_trad typically had better resolution and worse reliability. Reliability, unlike resolution, can be improved by appropriate adjustments, so these new methods potentially possess additional advantages compared to Cali_trad.

\section{e. Comparison of the various methods' skill}

Figure 6 compares BSs for the different methods, and shows that some methods outperform Cali_trad. The GSD and Ave_nbh scores are average values for the
10 members. The BS differences between the calibrated and uncalibrated methods are noticeable, and reflect the impacts of calibration even though only approximately 30 observed cases were used. Hence, if archived cases are not available originally, they can be gathered within a relatively brief period and used to improve forecasts [as also shown in Wilson and Vallée (2002)].

Figure 6 also shows that a reference score based on the simplified version of the method presented in Theis et al. (2005) yields a higher (i.e., worse) BS compared to the new approaches/methods. The binary forecasting method, computed through the use of a $1 \times 1$ neighborhood in the simplified Theis et al. (2005) method, yields a member-averaged BS of 0.1930, much higher than the other BSs computed.

\section{f. Sensitivity of results to grid spacing}

To evaluate the sensitivity of the methods to the grid spacing of the dataset, the most promising of the $20-\mathrm{km}$ methods were applied to an identical subdomain, but using the original unsmoothed 4-km grid spacing instead of the smoothed $20-\mathrm{km}$ spacing. The BSs for the methods improved with finer grid spacing at the 0.01-in. threshold (cf. Fig. 7 and Fig. 6) and the 0.10-in. threshold, but worsened slightly at the 0.25 -in. threshold. The differences in skill between methods, however, were similar at $4 \mathrm{~km}$ to what was indicated with the $20-\mathrm{km}$ results (Fig. 8). When applying the methods that use neighborhood approaches, the neighborhoods were scaled to fit with the $4-\mathrm{km}$ grid spacing (e.g., a $5 \times 5$ point area in the $20-\mathrm{km}$ results was replaced by a $25 \times 25$ point area in the $4-\mathrm{km}$ study). For this reason, there was a noticeable increase in computer resources and time required to verify the $4-\mathrm{km}$ forecasts. The $N \times N$

TABLE 6. Decomposed BSs, BSSs, bias scores, and ROC areas for the combination approach.

\begin{tabular}{|c|c|c|c|c|c|c|c|}
\hline \multirow[b]{2}{*}{ Threshold } & \multicolumn{7}{|c|}{ Score } \\
\hline & BS & Reliability & Resolution & Uncertainty & BSS & Bias & ROC \\
\hline 0.01 & 0.0959 & 0.0104 & 0.0601 & 0.1456 & 0.3411 & 1.2512 & 0.875 \\
\hline 0.10 & 0.0556 & 0.0066 & 0.0278 & 0.0767 & 0.2759 & 1.4126 & 0.903 \\
\hline 0.25 & 0.0340 & 0.0042 & 0.0131 & 0.0429 & 0.2083 & 1.6498 & 0.916 \\
\hline
\end{tabular}




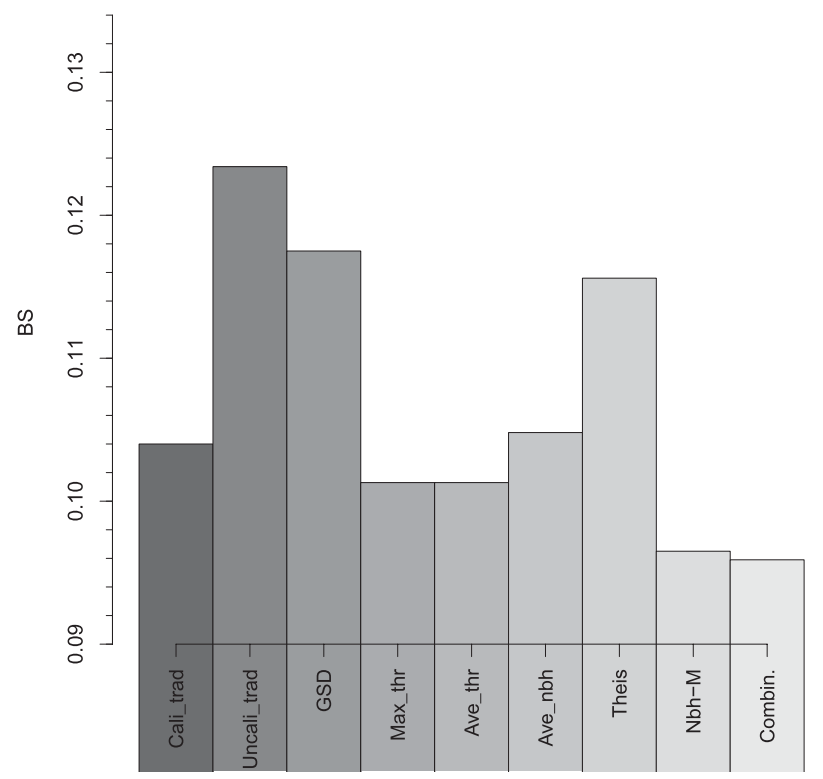

FIG. 6. The BSs for the 0.01-in. threshold for different methods at $20-\mathrm{km}$ grid spacing. See Table 1 for the notation used.

gridpoint neighborhoods with the best skill (lowest BSs) at $20 \mathrm{~km}$ also had the best skill at $4 \mathrm{~km}$.

Finding improved BSs at 4-km grid spacing compared to $20-\mathrm{km}$ grid spacing for the two-parameter point approach was unexpected because past deterministic studies have found that standard measures of skill usually show deteriorating skill as grid spacings are refined. Mass et al. (2002) and Gallus (2002) show that the equitable threat score (ETS) was higher when evaluating QPFs on coarser grid spacings compared to finer ones. However, these studies did not consider BSs, so it is unclear whether this statistic should follow the trends that ETS did. The 4-km decomposed BSs had lower uncertainty components than the $20-\mathrm{km}$ results at the 0.01 and 0.10 -in. thresholds, but not at the 0.25 -in. threshold (Table 7), so the improved 4-km results may be due to changes to the variability of observations in the dataset (i.e., the uncertainty). Comparisons of bias and ROC areas for the two grid spacings are also given in Table 7. The changes to the bias with increasing thresholds in the current study tended to agree with the Gallus (2002) Betts-Miller-Janjić (BMJ) control run bias comparisons. Bias was worse at finer grid spacings $[4 \mathrm{~km}$ here, $10 \mathrm{~km}$ in Gallus (2002)] than at coarser grid spacing [20 km here, $30 \mathrm{~km}$ in Gallus (2002)], but as the threshold increased the trend was reversed (Table 7). The ROC areas for Max_thr and Ave_thr remained in the 0.850.90 range for the 4-km results; however, Ave_thr had a decrease in ROC area from the 0.10 -in. threshold to the 0.25 -in. threshold, which did not exist in the $20-\mathrm{km}$ results (Table 7). Most of the 4-km grid-spacing ROC

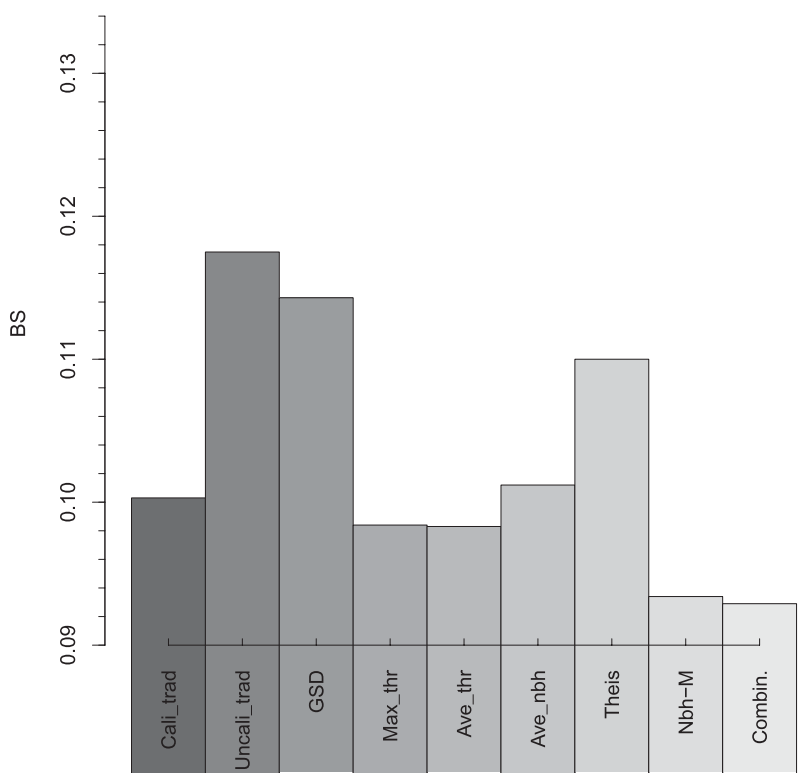

FIG. 7. As in Fig. 6, but for 4-km grid spacing.

areas were worse than the ROC areas for the $20-\mathrm{km}$ grid spacing, thus following the ETS trends shown in Gallus (2002) and Mass et al. (2002). Finally, a comparison of BSs for the combination approach and Cali_trad showed that statistically significant differences at the 99.9\% confidence level existed at all three thresholds for the $4-\mathrm{km}$ results, which was also the case for the $20-\mathrm{km}$ results.

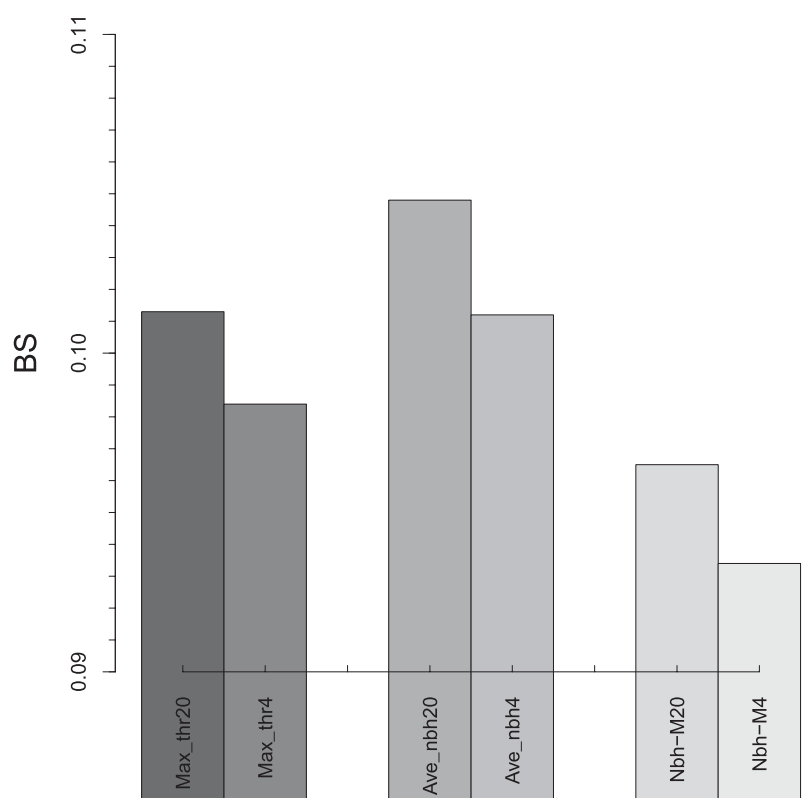

FIG. 8. Comparison of BSs for selected methods at 20- and 4-km grid spacing at the 0.01 -in. threshold. See Table 1 for the notation used. 
TABLE 7. Comparisons of bias, ROC areas, and uncertainty terms (of the decomposed BSs) from the Max_thr and Ave_thr methods between 20- and 4-km grid spacings at thresholds of $0.01,0.10$, and $0.25 \mathrm{in}$., with the better bias values and ROC areas for each grid spacing comparison shown in boldface.

\begin{tabular}{|c|c|c|c|c|c|c|c|}
\hline \multirow{2}{*}{ Grid $(\mathrm{km})$} & \multirow{2}{*}{ Threshold (in.) } & \multicolumn{2}{|c|}{ Bias } & \multicolumn{2}{|c|}{ ROC } & \multicolumn{2}{|c|}{ Uncertainty } \\
\hline & & Max_thr & Ave_thr & Max_thr & Ave_thr & Max_thr & Ave_thr \\
\hline \multirow[t]{3}{*}{20} & 0.01 & 1.2501 & 1.2501 & 0.857 & 0.862 & 0.1456 & 0.1456 \\
\hline & 0.10 & 1.4192 & 1.4405 & 0.877 & 0.865 & 0.0767 & 0.0767 \\
\hline & 0.25 & 1.6722 & 1.6972 & 0.897 & 0.869 & 0.0429 & 0.0429 \\
\hline \multirow[t]{3}{*}{4} & 0.01 & 1.2701 & 1.2692 & 0.853 & 0.852 & 0.1385 & 0.1385 \\
\hline & 0.10 & 1.4419 & 1.4602 & 0.883 & 0.853 & 0.0751 & 0.0751 \\
\hline & 0.25 & 1.6171 & 1.6227 & 0.890 & 0.839 & 0.0430 & 0.0430 \\
\hline
\end{tabular}

\section{Discussion and conclusions}

The present study is an extension of the single forecastbased POP approach used in GS04 and GBE07 to a 10-member WRF-ARW ensemble, while providing a comparison to a calibrated traditionally used equalweighting approach for determining POPs from ensembles. Exploratory tests were performed using a range of approaches, and some related variant methods were considered using data from early in the convection season in the central United States. The POPs were evaluated based on performance at each domain grid point. Quantification of the skill of the new approaches emphasized the use of BSs and ROC areas.

Because the approaches are based on postprocessing of simulated precipitation fields, tests were performed using both 20- and 4-km grid representations of the precipitation field. Hamill and Colucci (1997) showed that calibration over observations using a statistical approach improves forecasts, and the present paper found other approaches, also using observations, that improve forecasts. While the stage IV data used in this study have been noted to have biases relative to point observations, the calibration methodology used here could be used equally well to calibrate to point data.

For all methods, the most pronounced improvements in POP skill occurred for the lowest threshold, with diminishing improvements above a threshold of $0.25 \mathrm{in}$. Hence, the methods may be better at delineating areas experiencing precipitation and determining the location and timing of convective initiation compared to Cali_trad and Uncali_trad, more so than generating better forecasts of excessive rainfall.

By examining binned precipitation amounts and the number of ensemble members with precipitation greater than a threshold (the two-parameter point forecast approach), tabular POP forecasts, Max_thr and Ave_thr, were created. These methods had lower BSs than Cali_ trad (e.g., 0.1013 for Max_thr compared to 0.1040 for Cali_trad), and the differences between these methods and Cali_trad were statistically significant.
The two-parameter neighborhood approach [which, when its PAC binning and calibration are not applied, is conceptually similar to that of Theis et al. (2005)] provided skillful results that exceeded expectations. As discussed in Theis et al. (2005), the approach (consisting of the methods Max_nbh and Ave_nbh) is effectively an ensemble that is generated based on the spatial distribution of precipitation points in a neighborhood.

Ensembles generated using the neighborhood approach produced POPs as skillful as those from the 10member ensemble forecast Cali_trad. This suggests that the approach is very attractive operationally, and we are currently testing options to refine it in order to improve its performance. Because postprocessing of a single deterministic simulation can provide skill comparable to that obtained by Cali_trad, computer resources used for the traditional ensemble simulation might be better used for further refinement of the model grid spacing or for improved model physical formulation. A replacement for the traditional ensemble information can be obtained from POPs using postprocessing to generate spatially based ensembles. It is possible, however, that a traditionally calibrated ensemble with more than 10 members (used in the present study) or an ensemble with different design characteristics may yield better probabilistic forecasts than that based on the POP of a single member.

A limited comparison of skill between 20- and 4-km gridded precipitation POP forecasts indicated better BSs for the 4-km setting. While this pattern needs to be further evaluated in future studies, it appears to support the use of fine grid resolution single runs versus coarse grid ensembles. Questions remain about the best usage of computer resources for predicting convective QPFs. For instance, is it better to run a single deterministic refined grid simulation or a coarser grid ensemble? Both deterministic and probabilistic forecasts were more accurate for the 4-km grid spacing with explicit convection, and Clark et al. (2009) found that models run on finer grid spacings tended to provide more accurate forecasts than forecasts on coarser grid spacings. A similar 
conclusion was reached by Roberts and Lean (2008) using FSS. Faster error growth at finer grid spacings in the Clark et al. (2009) study led to increased spread and more reliable forecasts.

A two-parameter neighborhood- $M$ approach considered binned PAC and ensemble member agreements (in the 10-member model ensemble and the neighborhood ensemble) with precipitation greater than a threshold, and produced POP forecasts of even higher skill than the two-parameter point forecast approach and the twoparameter neighborhood approach. When all three approaches were considered together with Cali_trad, the resulting combination approach produced forecasts that were statistically significantly better compared to Cali_ trad's forecasts at the $99.9 \%$ confidence level with $p$ values that were nearly 0 at all thresholds.

Overall, this study suggests that three of its evaluation techniques potentially can be used to provide useful POP forecasts. Two of the evaluation techniques are represented by the two general parameters used within the approaches: binning a PAC and determining the member agreement percentage. The PAC-binning parameter was used in all of the new approaches, as well as in GSD. In this study, the benefits of the PAC-binning parameter were especially apparent when considering the ROC areas for the new approaches introduced and GSD, because these ROC areas increased further than the ROC areas for Cali_trad and Uncali_trad. The member agreement percentage parameter is used in Cali_trad and Uncali_trad in addition to the approaches introduced in this study because it is well established as an important POP-forecasting technique. The third evaluation technique that can provide useful POP forecasts is the neighborhood approach. We showed that using a neighborhood of grid points can yield probabilistic information from deterministic forecasts, produce POP forecasts that may rival traditional calibrated ensemble POP forecasts, and also improve traditional ensemble forecasts.

The new approaches evaluated in the present study provide a significant extension and improvement to the one-parameter GSD approach evaluated in GS04 and GBE07. Also, the new research suggests that improved POPs are related more to an increased averaged precipitation amount in the surroundings of an evaluation domain's grid points, rather than an increased precipitation amount at the grid point itself, as was assumed in these two prior studies.

Additional research is needed, first, to examine and further improve the well-performing approaches, particularly the neighborhood-related ones. For example, sensitivities to the simulated domain location and size, as well as the time of day, should be examined. Special attention should be given to the sensitivity of neighborhood approaches to terrain features that are associated with convective forcing, such as elevated regions or sea-land variations, where the degree of areal-temporal randomness in precipitation is constrained. A weighted neighborhood approach could possibly be used to more realistically handle local terrain effects. Second, research is also needed to compare on an event-by-event basis the performance of various relevant approaches for precipitation forecasts. This comparison should be beneficial in obtaining insight conducive to further improvements in the approaches.

The research for this study began at a time when only the 2007 and 2008 Spring Experiment output was available. Any future work related to these approaches may consider incorporating the 2009 and 2010 Spring Experiment data, as there would be several advantages to doing so. There was evidence to suggest that some neighborhood approaches, which created a very large number of POP forecast scenarios, may have been limited by a lack of data within certain bins. Adding the 2009 and 2010 data could provide more data for the correct alarm ratio calculations, and lead to more accurate POPs. Future work should also compare the performance of the approaches to that of the MOS approach currently used operationally in the United States.

Acknowledgments. This research was funded in part by National Science Foundation Grants ATM-0537043 and ATM-0848200, with funds from the American Recovery and Reinvestment Act of 2009. The authors thank Adam Clark for providing the model precipitation output and for helpful discussions, Jon Hobbs for his advice regarding the statistical analysis, and Daryl Herzmann for his computational assistance. We appreciate the constructive comments of Elizabeth Ebert and two anonymous reviewers that improved the paper.

\section{APPENDIX}

\section{Computation of POPs in Neighborhoods Truncated by Domain Boundaries}

When a neighborhood of areal squares extended outside the domain used in this study, the value $F_{1}$ of the parameter(s) from the neighborhood was extrapolated using the following equation:

$$
F_{1}=\frac{F_{o} \times A_{t}}{A},
$$

where $F_{0}$ is the number of forecasts within the neighborhood with precipitation greater than the threshold, 
$A_{t}$ is the number of points that should exist in the neighborhood, and $A$ is the number of points that actually exist within a given neighborhood. If the neighborhood is entirely within the domain, then $A_{t}=A$, so $F_{0}=F_{1}$. If the neighborhood is partially outside of the domain, then $A<A_{t}$, and Eq. (A1) will approximate what agreement the neighborhood would likely have had if an entire neighborhood were considered. With the agreement parameter determined, the POP would then be calculated according to the approach's specifications.

\section{REFERENCES}

Baldwin, M. E., and K. E. Mitchell, 1997: The NCEP hourly multisensory U.S. precipitation analysis for operations and GCIP research. Preprints, 13th Conf. on Hydrology, Long Beach, CA, Amer. Meteor. Soc., 54-55.

Buizza, R., A. Hollingsworth, F. Lalaurette, and A. Ghelli, 1999: Probabilistic predictions of precipitation using the ECMWF Ensemble Prediction System. Wea. Forecasting, 14, 168-189.

Chakraborty, A., and T. N. Krishnamurti, 2006: Improved seasonal climate forecasts of the South Asian summer monsoon using a suite of 13 coupled ocean-atmosphere models. Mon. Wea. Rev., 134, 1697-1721.

Clark, A. J., W. A. Gallus Jr., M. Xue, and F. Kong, 2009: A comparison of precipitation forecast skill between small convectionallowing and large convection-parameterizing ensembles. Wea. Forecasting, 24, 1121-1140.

Ebert, E. E., 2001: Ability of a poor man's ensemble to predict the probability and distribution of precipitation. Mon. Wea. Rev., 129, 2461-2480.

- 2008: Fuzzy verification of high resolution gridded forecasts: A review and proposed framework. Meteor. Appl., 15, 51-64.

$\longrightarrow$, 2009: Neighborhood verification: A strategy for rewarding close forecasts. Wea. Forecasting, 24, 1498-1510.

Gallus, W. A., Jr., 2002: Impact of verification grid-box size on warmseason QPF skill measures. Wea. Forecasting, 17, 1296-1302.

— rainfall indicate enhanced likelihood of rain occurrence? Wea. Forecasting, 19, 1127-1135.

— - M. E. Baldwin, and K. L. Elmore, 2007: Evaluation of probabilistic precipitation forecasts determined from Eta and AVN forecasted amounts. Wea. Forecasting, 22, 207-215.

Gilleland, E., D. Ahijevych, B. G. Brown, B. Casati, and E. E. Ebert, 2009: Intercomparison of spatial forecast verification methods. Wea. Forecasting, 24, 1416-1430.

Hamill, T. M., and S. J. Colucci, 1997: Verification of Eta-RSM short-range ensemble forecasts. Mon. Wea. Rev., 125, 1312-1327.
— , and J. S. Whitaker, 2006: Probabilistic quantitative precipitation forecasts based on reforecast analogs: Theory and application. Mon. Wea. Rev., 134, 3209-3229.

Kong, F., and Coauthors, 2007: Preliminary analysis on the realtime storm-scale ensemble forecasts produced as a part of the NOAA Hazardous Weather Testbed 2007 Spring Experiment. Preprints, 22nd Conf. on Weather Analysis and Forecasting/ 18th Conf. on Numerical Weather Prediction, Park City, UT, Amer. Meteor. Soc., 3B.2. [Available online at http://ams. confex.com/ams/pdfpapers/124667.pdf.]

Leith, C. E., 1974: Theoretical skill of Monte Carlo forecasts. Mon Wea. Rev., 102, 409-418.

Mass, C. F., D. Ovens, K. Westrick, and B. A. Colle, 2002: Does increasing horizontal resolution produce more skillful forecasts? Bull. Amer. Meteor. Soc., 83, 407-430.

Murphy, A. H., 1973: A new vector partition of the probability score. J. Appl. Meteor., 12, 595-600.

Roberts, N. M., and H. W. Lean, 2008: Scale-selective verification of rainfall accumulations from high-resolution forecasts of convective events. Mon. Wea. Rev., 136, 78-97.

Schwartz, B. E., and S. G. Benjamin, 2000: Verification of RUC2 precipitation forecasts using the NCEP multisensory analysis. Preprints, Fourth Symp. on Integrated Observing Systems, Long Beach, CA, Amer. Meteor. Soc., 182-185.

Schwartz, C. S., and Coauthors, 2010: Toward improved convectionallowing ensembles: Model physics sensitivities and optimizing probabilistic guidance with small ensemble membership. Wea Forecasting, 25, 263-280.

Skamarock, W. C., 2004: Evaluating mesoscale NWP models using kinetic energy spectra. Mon. Wea. Rev., 132, 3019-3032.

Theis, S. E., A. Hense, and U. Damrath, 2005: Probabilistic precipitation forecasts from a deterministic model: A pragmatic approach. Meteor. Appl., 12, 257-268.

Tustison, B., D. Harris, and E. Foufoula-Georgiou, 2001: Scale issues in verification of precipitation forecasts. J. Geophys. Res., 106, $11775-11784$.

Wilks, D. S., 2006: Statistical Methods in the Atmospheric Sciences 2nd ed. Academic Press, 627 pp.

Wilson, L. J., and M. Vallée, 2002: The Canadian Updateable Model Output Statistics (UMOS) system: Design and development tests. Wea. Forecasting, 17, 206-222.

Xue, M., and Coauthors, 2008: CAPS realtime storm-scale ensemble and high-resolution forecasts as part of the NOAA Hazardous Weather Testbed 2008 Spring Experiment. Preprints, 24th Conf. on Severe Local Storms, Savannah, GA, Amer. Meteor. Soc., 12.2. [Available online at http://ams. confex.com/ams/pdfpapers/142036.pdf.]

Yussouf, N., and D. J. Stensrud, 2008: Reliable probabilistic quantitative precipitation forecasts from a short-range ensemble forecasting system during the 2005/06 cool season. Mon. Wea Rev., 136, 2157-2172. 